reduction effectiveness, the cost-benefit evaluation and the analytic background. While the development and evaluation of the measures will be developed into a format and structure that will enable industry, policy-makers and other stakeholders to access the information in an efficient manner within the DSS.

In order to provide policy-makers and industry with comprehensive and well-structured information about measures, it is essential that a systems approach is used to ensure the links between risk factors and all relevant safety measures are made fully visible. The DSS is intended to become a major source of information for industry, policy-makers and the wider road safety community; it will incorporate the knowledge base of accident causation, risks and measures that will be developed in the project and the underlying methodological systems. It will enable a considerable advance in the provision of evidence-based road safety policies. It will be developed in a form that can readily be incorporated within the existing European Road Safety Observatory of the European Commission DG-MOVE.

Methods For the development of the European Road Safety Decision Support System a comprehensive common methodology is designed and applied in existing and new studies of road safety measures effectiveness evaluation. The DSS covers all types of road safety interventions, including the road user behaviour, infrastructure, vehicle, as well as road safety management.

Results In this paper, the structure and the functioning of the European Road Safety Decision Support System will be presented, together with the first results of the application of the common methodology for the evaluation of road safety measures effectiveness.

Conclusions The development of the European Road Safety Decision Support System presents a great potential to further support decision making at local, regional, national and international level, aiming to fill in the current gap of comparable measures effectiveness evaluation across Europe and worldwide.

\section{POLICYMAKER PERSPECTIVES ON WHY EVIDENCE- BASED TRAFFIC SAFETY POLICIES ARE SUPPORTED OR OPPOSED}

Deborah C Girasek. Uniformed Services University of the Health Sciences, USA

\subsection{6/injuryprev-2016-042156.81}

Background The Centres for Disease Control and Prevention have recognised the United States' reduction in motor vehicle fatalities as one of the $20^{\text {th }}$ century's greatest public health achievements. Public policy played a critical role in this progress. Regulatory oversight and public investment yielded safer vehicles and modern highways. State legislation was passed to encourage members of the public to drive safely and use protective equipment. Many jurisdictions however, both within the United States and internationally, are not benefitting from policies that have been shown to save lives. The purpose of this study was to increase our understanding of the barriers that prevent legislators from adopting evidence-based traffic safety policies.

Methods A review of legislative activity was carried in the 2013 and 2014 to identify traffic safety bills under consideration at state legislatures within the United States. Four bills were selected for study inclusion because there was scientific evidence that suggests they would likely have influenced injury outcomes, and they had received mixed votes within the state's legislative body. Legislators who had voted for and against the bills were invited to participate in semi-structured interviews. Discussions were audiotaped, and subsequently transcribed. They were then coded for themes. A sample of the data was also coded by a second reviewer, to ensure that a reliable coding scheme had been developed.

Results The characteristics of legislators who agreed and declined to participate in the study will be reported. The perspectives of policymakers who voted for and against the proposed safety laws will be contrasted, with regard to issue salience, factors influencing their vote, whether interest groups or constituents contacted them and what they believed that the consequences of the bill's passage would have been. General legislative insights into what safety advocates need to understand about a policymaker's perspective will also be presented.

Conclusions Many factors other than scientific evidence influence whether legislators support important safety legislation. More research is needed into how to overcome value-driven objections. Safety advocates would benefit from a more sophisticated understanding of the legislative process, and/or partnering with professionals who are more familiar with the policymaking environment.

\section{SAFE ROADS || SAFE KIDS: PUBLIC PRIVATE PARTNERSHIPS TO INCREASE GLOBAL ROAD SAFETY FOR CHILDREN}

${ }^{1}$ Shannon O Sullivan, ${ }^{2}$ Shane O'Connor. ${ }^{1}$ Safe Kids Worldwide; ${ }^{2}$ FedEx

10.1136/injuryprev-2016-042156.82

Background More than 500 children die each day from roadrelated injuries - 186,000 annually. By harnessing the reach, reputation and responsibility of corporations working with governments, NGOs and civil society, Safe Roads || Safe Kids demonstrates the power of public-private partnerships to elevate awaress of road safety for children and hasten adoption of changes to protect lives.

Description of the problem While high-income countries are experiencing declines in deaths from road injuries, death rates are rising in 68 countries. Children living in poorer nations are most at risk. More than $90 \%$ of child road deaths occur in low and middle-income countries. By 2030, deaths from road crashes are expected to surpass deaths from HIV/AIDS, malaria and other infectious diseases. And it is our most vulnerable road users children - who are most at risk. This is a tragedy that we can predict and prevent.

Results

- In its 15 th year, Safe Kids Worldwide's Walk this Way program, sponsored by FedEx, supported pedestrian safety in 10 countries, educating 1,298,000 students in 2,893 schools.

- In 2014, Safe Kids, FedEx, and partners launched Safe Roads | Safe Kids to focus attention, awareness and resources on road safety for children.

- In May 2015, Safe Kids, with support from FedEx, celebrated Global Road Safety Week, coordinating 300 events across 89 cities in 17 countries, drawing more than 46,000 participants. To date, the \#SaveKidsLives campaign has 900,000 signatures.

- To date, Safe Roads I| Safe Kids has resulted in new corporate support (Denso, BG Group), joining long-standing commitments from FedEx and GM Foundation.

Conclusions If we hope to meet the goal of saving 5 million lives by 2020 as set forth by the UN Decade of Action for Road Safety, we need a clear plan of action and a broad coalition poised for 